\section{Lightning-Induced Voltages in a Satellite Launch-Pad Protection System}

\author{
P. Durai Kannu and M. Joy Thomas
}

\begin{abstract}
Modern satellite launch pads are provided with lightning protection systems to take care of direct lightning strike to the satellite launch vehicle during the launch preparation period. This paper presents the results of the study on induced voltage on the conductors interlinking the towers of such a protection system due to a nearby lightning strike. The variation in the induced voltages with lightning-strike distances as well as ground conductivities are studied and the results presented in the paper. It has been seen that the waveshape of the induced voltage depends on conductor height and striking distance, whereas the ground conductivity has negligible effect on the induced voltage.
\end{abstract}

Index Terms-Electromagnetic field coupling, ground conductivity, induced voltages, lightning protection, satellite launch pad.

\section{INTRODUCTION}

Lightning poses a serious threat to tall objects. A satellite launch system is a tall structure standing over a flat terrain, and hence it is necessary to prevent it from direct lightning strikes by diverting the lightning strike to a close by relatively safe spot [1]. All modern satellite launch pads are provided with a lightning protection system, in order to protect the satellite launch vehicle from direct lightning strikes.

The launch-pad protection system under study consists of three towers, each $120 \mathrm{~m}$ in height, which are located at the vertices of an equilateral triangle of base $180 \mathrm{~m}$. There are five conductors connecting the tower tops. All the five conductors are bundled at regular intervals. A simplified diagram of the satellite launch-pad protection system is shown in Fig. 1. Any lightning strike coming close to the launch pad is attracted to the tall towers or the conductors interlinking them and gets diverted to the ground, thereby avoiding a direct strike to the launch vehicle. However, a nearby lightning strike can induce very high voltage on the lightning protection system conductors as well as the lighting cables on the towers, by induction. It also becomes necessary to characterize the induced voltage waveform due to an indirect strike so that the lightning-strike monitoring system of the protection scheme can discriminate between a direct strike and an indirect strike, and this has been the main motivation for taking up this work. The results presented in this paper are also useful in computing the induced voltages on transmission line conductors operating at ultrahigh voltage (UHV) levels, as the tower height for such systems is comparable to heights of the towers described in this paper.

Over voltages induced on an overhead conductor due to a nearby lightning strike is generally performed by: 1) calculating the electromagnetic field produced by the lightning return-stroke by making use of an appropriate return-stroke model, and 2) computing the coupling of these fields to the conductors by means of a suitable coupling model and thereby the estimation of induced voltage. The ground conductivity influences the magnitude and waveshape of the radiated lightning fields as well as the surge propagation along the line. The induced voltage is dependent on the conductor height as well as the lightning striking distance. This paper gives the results of the induced voltages computed at the conductor midspan and on the tower top due to a nearby lightning strike. The effect of ground conductivity as well as the lightning striking distance on the induced voltage are also discussed.

Manuscript received September 10, 2002; revised Februray 5, 2003.

The authors are with the Department of High Voltage Engineering, Indian Institute of Science, Bangalore 560012, India.

Digital Object Identifier 10.1109/TEMC.2003.819064

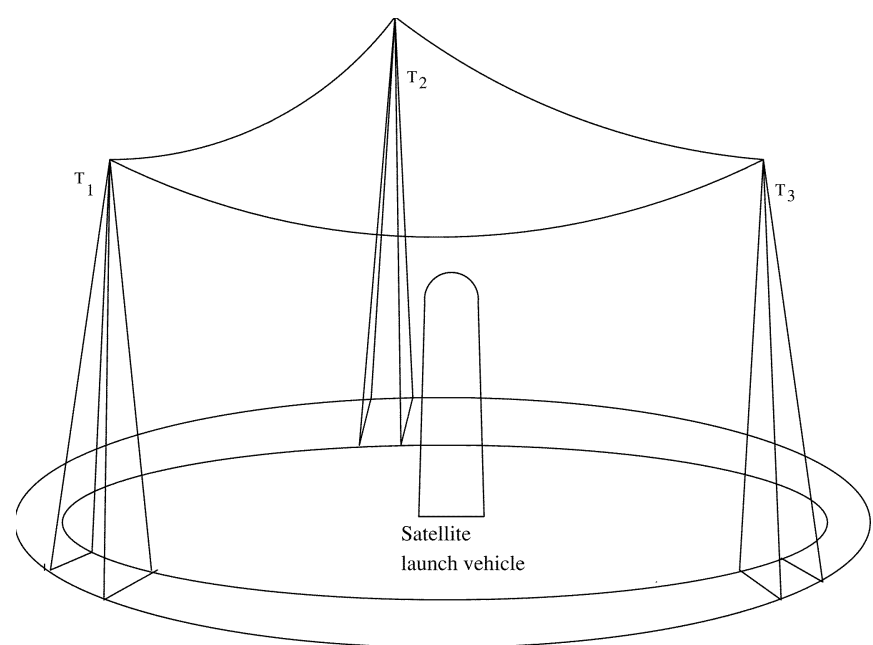

Fig. 1. Simplified diagram of launching pad protection system. The $T$ 's are the three towers.

\section{LightNing ELECTROMAGNETIC FIELDS}

For the calculation of the electromagnetic field, the lightning return-stroke channel is assumed to be straight and vertical. The modified transmission-line (MTL) model [2] has been used for the representation of the return-stroke channel current. According to this model, the lightning current is allowed to decrease with height while propagating along the channel upward. The return-stroke current $i\left(z^{\prime}, t\right)$ at height $z^{\prime}$ and at time $t$ is represented as

$$
i\left(z^{\prime}, t\right)=e^{\left(-\frac{z^{\prime}}{\lambda}\right)} i\left(0, t-\frac{z^{\prime}}{v}\right)
$$

where $v$ is the velocity of the return stroke and $\lambda$ is the decay constant to account for the effect of the vertical distribution of the charge stored in the corona sheath of the leader and subsequent discharge during the return-stroke phase.

The return-stroke current at the ground strike point $i(0, t)$, can be described by a double exponential expression [3]

$$
i(0, t)=i_{0}\left(e^{-\alpha t}-e^{-\beta t}\right) .
$$

For a peak lightning-stroke current of $\simeq 10 \mathrm{kA}$ at the ground level, the values of $i_{0}, \alpha$, and $\beta$ are chosen as $10 \mathrm{kA}, 3 \times 10^{8} \mathrm{~s}^{-1}$ and $10^{7} \mathrm{~s}^{-1}$, respectively [3]. The return-stroke velocity $v$ is taken as $1.1 \times 10^{8} \mathrm{~m} / \mathrm{s}$ and decay constant $\lambda$ is taken as $1.7 \mathrm{~km} \mathrm{[4].}$

From the lightning channel at an arbitrary point $P(r, \phi, z)$ which is at a distance of $r$ and height $z$, the vertical electric field $d E_{z}(r, \phi, z)$ and horizontal electric field $d E_{r}(r, \phi, z)$ produced by a current element of length $d z^{\prime}$ is given by the following expressions in time domain [4]-[6]. These expressions are valid when the ground is assumed as a perfect conductor and for the MTL model of the return stroke

$$
\begin{gathered}
d E_{z}(r, z, t)=\frac{d z^{\prime}}{4 \pi \epsilon_{0}}\left[\frac{2\left(z-z^{\prime}\right)^{2}-r^{2}}{R^{5}} e^{\left(-\frac{z^{\prime}}{\lambda}\right)} \int_{0}^{t} i\left(\frac{0, \tau-z^{\prime}}{v}-\frac{R}{c}\right) d \tau\right. \\
+\frac{2\left(z-z^{\prime}\right)^{2}-r^{2}}{c R^{4}} e^{\left(-\frac{z^{\prime}}{\lambda}\right) i\left(\frac{0, \tau-z^{\prime}}{v}-\frac{R}{c}\right)} \\
\left.-\frac{r^{2}}{c^{2} R^{3}} e^{\left(-\frac{z^{\prime}}{\lambda}\right)} \frac{\partial}{\partial t} i\left(0, t-\frac{z^{\prime}}{v}-\frac{R}{c}\right)\right]
\end{gathered}
$$




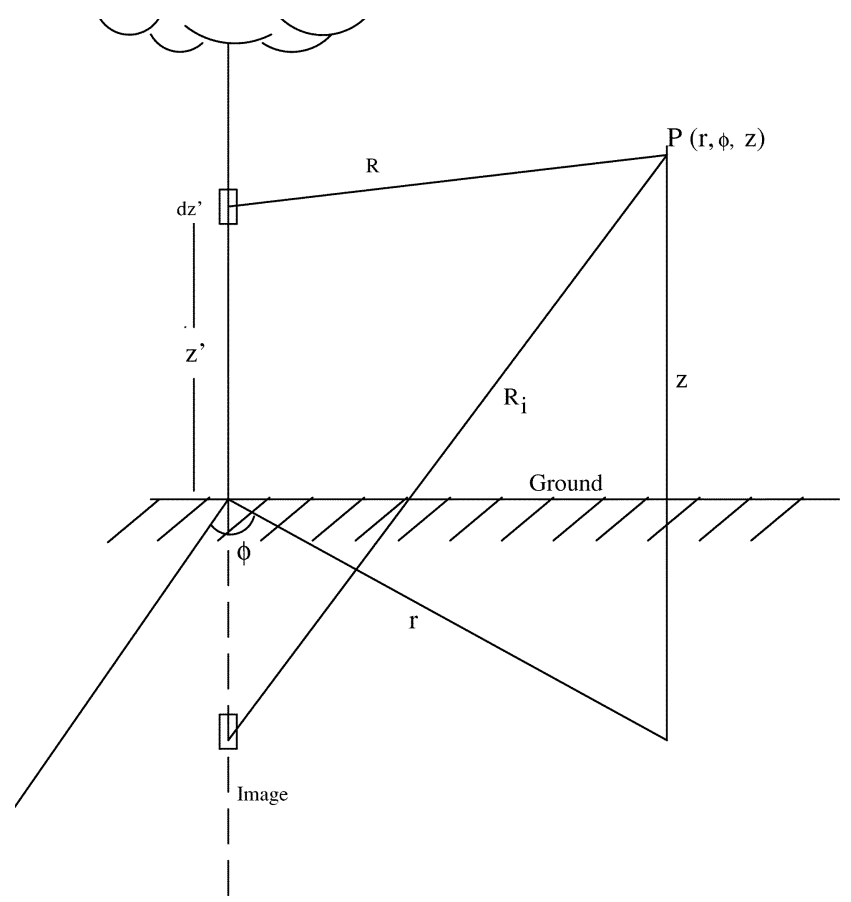

Fig. 2. Sketch showing the lightning channel and its image used in calculating the return-stroke fields.

$$
\begin{aligned}
d E_{r}(r, z, t)=\frac{d z^{\prime}}{4 \pi \epsilon_{0}} & {\left[\frac{3 r\left(z-z^{\prime}\right)}{R^{5}} e^{\left(-\frac{z^{\prime}}{\lambda}\right)} \int_{0}^{t} i\left(\frac{0, \tau-z^{\prime}}{v}-\frac{R}{c}\right) d \tau\right.} \\
+ & \frac{3 r\left(z-z^{\prime}\right)}{c R^{4}} e^{\left(-\frac{z^{\prime}}{\lambda}\right)} i\left(\frac{0, \tau-z^{\prime}}{v}-\frac{R}{c}\right) \\
+ & \left.\frac{r\left(z-z^{\prime}\right)}{c^{3} R^{3}} e^{\left(-\frac{z^{\prime}}{\lambda}\right)} \frac{\partial}{\partial t} i\left(0, t-\frac{z^{\prime}}{v}-\frac{R}{c}\right)\right]
\end{aligned}
$$

where $\epsilon_{0}$ is the permittivity of free space and $c$ is the velocity of light. $R=\sqrt{r^{2}+\left(z-z^{\prime}\right)^{2}}$ is the distance from the current element to the observation point. The effects of a perfectly conducting ground plane on the electromagnetic fields generated by the current element is included by replacing the ground by an image current at a distance $z^{\prime}$ below the ground. A sketch showing the lightning channel and its image used in calculating the return-stroke fields is shown in Fig. 2.

It has been reported that ground conductivity influences the horizontal electric field radiated by lightning [4] and hence it was decided to study the effect of finite ground conductivity on the electric field and thereby the induced voltages on conductors as well as the tower top which are at a very large height from the ground. The horizontal electric field including the ground conductivity can be calculated using Cooray-Rubinstien formula [7]. This formula requires the magnetic field produced by the return stroke. The magnetic field $d H_{\phi}(r, \phi, z)$ due to the current element $d z^{\prime}$ using the MTL model for the return stroke is given by

$$
\begin{aligned}
d H_{\phi}(r, z, t)= & \frac{d z^{\prime}}{4 \pi}\left[\frac{r}{c R^{2}} e^{\left(-\frac{z^{\prime}}{\lambda}\right)} \frac{\partial}{\partial t} i\left(\frac{0, t-z^{\prime}}{v}-\frac{R}{c}\right)\right. \\
& \left.+\frac{r}{R^{3}} e^{\left(-\frac{z^{\prime}}{\lambda}\right)} i\left(\frac{0, t-z^{\prime}}{v}-\frac{R}{c}\right)\right]
\end{aligned}
$$

where $\mu_{0}$ and $\sigma_{g}$ are the permeability of free space, and the conductivity of the ground, respectively. The total vertical and horizontal electric fields as well as the magnetic field are obtained by integrating (3)-(5) along the channel and its image.

The horizontal electric field with the finite ground conductivity $E_{r g}(z=h, r)$ is given as [7]

$$
E_{r g}(z=h, r)=E_{r}(z=h, r)-H_{\phi}(z=0, r) \frac{\sqrt{\mu_{0}}}{\sqrt{\epsilon+\frac{\sigma_{g}}{j \omega}}}
$$

where $E_{r}(z=h, r)$ is the Fourier transform of the horizontal electric field at height $h, H_{\phi}(z=0, r)$ is the Fourier transform of the azimuthal component of the magnetic field at ground level. $\mu_{0}$ is the permeability of air, $\epsilon$ and $\sigma_{g}$ are the permittivity and conductivity of the ground, respectively. Both $E_{r}(z=h, r)$ and $H_{\phi}(z=0, r)$ are calculated assuming a perfect conducting ground using (4) and (5).

\section{Calculation of Induced Voltage}

\section{A. The Coupling Model}

For the coupling of field to overhead conductors, a set of time-domain equations describing the voltages induced on multiconductors as proposed by Agrawal et al. [8] have been used. The frequency-dependent-series ground impedance is represented in the time-domain coupling equation by a convolution integral [9]. Hence, for the conductors along the $x$ axis, the set of differential equations describing the coupling of the electromagnetic field with the multiconductors above the ground are expressed as

$$
\begin{aligned}
\frac{\partial}{\partial x}\left[v_{i}^{s}(x, t)\right]+\left[L_{i j}\right] \frac{\partial}{\partial t}\left[i_{i}(x, t)\right] & \\
+\int_{0}^{t}\left[\xi_{i j}(t-\tau)\right] \frac{\partial}{\partial \tau}\left[i_{i}(x, \tau)\right] d \tau & =\left[E_{x}^{i}\left(x, h_{i}, t\right)\right] \\
\frac{\partial}{\partial x}\left[i_{i}(x, t)\right]+\left[G_{i j}\right]\left[v_{i}^{s}(x, t)\right] & \\
+\left[C_{i j}\right] \frac{\partial}{\partial t}\left[v_{i}^{s}(x, t)\right] & =0
\end{aligned}
$$

where the vector $\left[E_{x}^{i}\left(x, h_{i}, t\right)\right]$ is the horizontal component of the incident electric field along the conductor at conductor height $h_{i}$. The subindex $i$ denotes the particular wire of the multiconductor line. $\left[L_{i j}\right]$, $\left[G_{i j}\right]$, and $\left[C_{i j}\right]$ are the inductance, conductance, and capacitance matrices per unit length of the line, respectively. In this paper, the mutual coupling between the conductor spans are not considered. $\left[i_{i}\right]$ is the line current vector. $\left[\xi_{i j}\right]$ is the transient ground resistance matrix and is equal to the inverse Fourier transform of $\left[Z_{g_{i j}} / j \omega\right]$, ie., $\xi_{i j}(t)=$ $F^{-1}\left[\left(Z_{g_{i j}}\right) / j \omega\right]$ and $\left[Z_{g_{i j}}\right]$ is the ground impedance matrix. The internal impedance of the line is neglected. $\left[v_{i}^{s}\right]$ is the scattered voltage vector on the $i$ th conductor. This is the voltage due to the field produced by the induced currents on the conductors. The scattered voltage is related to the total line voltage $v_{i}(x, t)$ by the following relation:

$$
\left[v_{i}(x, t)\right]=\left[v_{i}^{s}(x, t)\right]+\left[v_{i}^{i}(x, t)\right]
$$

where $\left[v_{i}^{i}(x, t)\right]$ is the incident voltage

$$
\left[v_{i}^{i}(x, t)\right]=-\int_{0}^{h_{i}} E_{z}^{i}(x, z, t) d z
$$

where $E_{z}^{i}(x, z, t)$ is the incident (or inducing) vertical electric field at $x$ at a height of $z$.

The voltage at the end of the line is determined by the boundary condition and the current at the two ends of the line, viz., $i\left(x_{0}, t\right)$ and $i\left(x_{0}+l, t\right)$, where $l$ is the span of the overhead line. The boundary conditions for the scattered voltage are

$$
\begin{gathered}
v_{i}^{s}\left(x_{0}, t\right)=-\left[Z_{1}\right]\left[i_{i}\left(x_{0}, t\right)\right]+\int_{0}^{h_{i}} E_{z}^{i}\left(x_{0}, z, t\right) d z \\
v_{i}^{s}\left(x_{0}+l, t\right)=\left[Z_{2}\right]\left[i_{i}\left(x_{0}+l, t\right)\right]+\int_{0}^{h_{i}} E_{z}^{i}\left(x_{0}+l, z, t\right) d z
\end{gathered}
$$

where $\left[Z_{1}\right]$ and $\left[Z_{2}\right]$ are the terminating impedance matrices. Fig. 3 shows the equivalent circuit of a single wire overhead line excited by the lightning return-stroke electromagnetic field. 


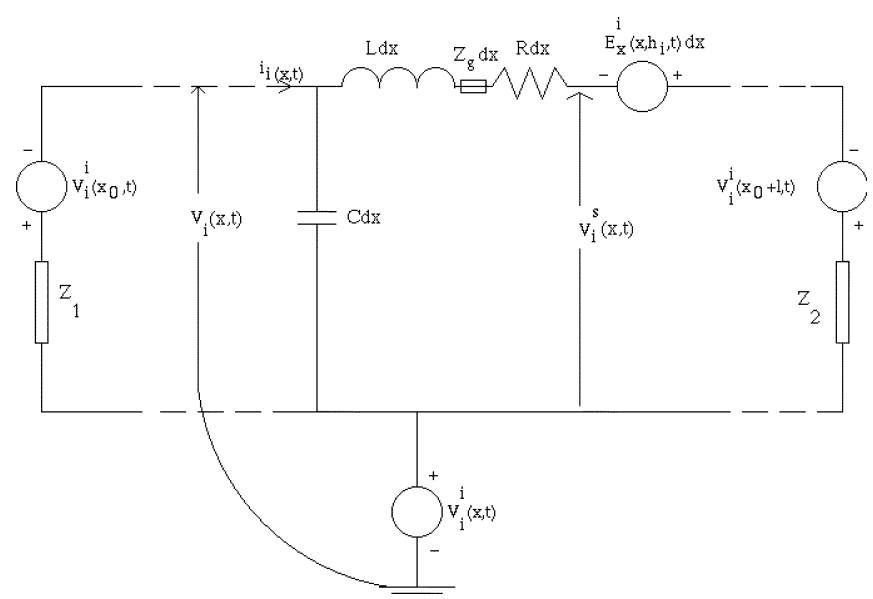

Fig. 3. Equivalent circuit of a single-wire overhead line excited by lightning return-stroke electromagnetic field. Conductance is neglected.

\section{B. Transient Ground Resistance}

The expression for the mutual ground impedance between the conductors in low-frequency approximation, ie., assuming $\sigma_{g} \gg \omega \epsilon_{0} \epsilon_{r}$ is given by [10]

$$
Z_{g_{i j}}=\frac{j \omega \mu_{0}}{\pi} \int_{0}^{\infty} \frac{e^{-\left(h_{i}+h_{j}\right) x} \cos \left(r_{i j} x\right)}{\sqrt{x^{2}+j \omega \mu_{0} \sigma_{g}}+x} d x
$$

where $r_{i j}$ is the distance between the two conductors in the horizontal plane.

The general expression for the ground-impedance matrix does not have an analytical inverse Fourier transform. However, a low-frequency approximation of the inverse Fourier transform of the ground impedance is given by [11]

$$
\begin{aligned}
\xi_{i j}(t)=\frac{\mu_{0}}{\pi T_{i j}}[ & \frac{1}{2 \sqrt{\pi}} \sqrt{\frac{T_{i j}}{t}} \cos \left(\frac{\theta_{i j}}{2}\right)+\frac{1}{4} e^{\frac{T_{i j} \cos \left(\theta_{i j}\right)}{t}} \\
& \times \cos \left(\frac{T_{i j}}{t} \sin \left(\theta_{i j}\right)-\theta_{i j}\right)-\frac{1}{2 \sqrt{\pi}} \\
& \times \sum_{n=0}^{\infty} a_{n}\left(\frac{T_{i j}}{t}\right)^{\frac{2 n+1}{2}} \cos \left(\frac{2 n-1}{2} \theta_{i j}\right) \\
& \left.-\frac{\cos \left(\theta_{i j}\right)}{4}\right]
\end{aligned}
$$

where $\tau_{i i}=h_{i}^{2} \mu_{0} \sigma_{g}$ and $a_{n}=\left(2^{n}\right) /(1.3 \ldots(2 n+1))$ with

$$
\left[\frac{h_{i}+h_{j}}{2}+j \frac{r_{i j}}{2}\right]^{2} \mu_{0} \sigma_{g}=T_{i j} e^{j \theta_{i j}} .
$$

In (14), if $h_{i}=h_{j}$, and $r_{i j}=0$, then, $\theta_{i j}=0$ and we get the expression for diagonal terms of $\left[\xi_{i j}(t)\right]$.

The elements of ground transient resistance matrix $\left[\xi_{i j}(t)\right]$ presents singularity as $t \rightarrow 0$, as

$$
\operatorname{Lim}_{t \rightarrow 0} \xi_{i j}(t) \simeq \frac{\mu_{0}}{2 \pi} \sqrt{\frac{1}{\pi T_{i j} t}} \cos \left(\frac{\theta_{i j}}{2}\right) .
$$

In (15), $\xi_{i j}(t)$ tends to infinity when $\mathrm{t}=0$. However, Loyka [12] has shown that if $\xi_{i j}(t)$ is calculated without the low-frequency approximation, it will tend to be a constant as $t \rightarrow 0$. In this paper, $\xi_{i j}(t)$ is calculated using (14) and (15) for $t>0.1 T_{i j}$ and $t<0.1 T_{i j}$, respectively. In solving the convolution integral, the ground transient resistance is taken as a constant in the range $t-\Delta t<\tau<t$. Hence, for the calculation of line current at the $n$th incremental time $t=n \Delta t$,

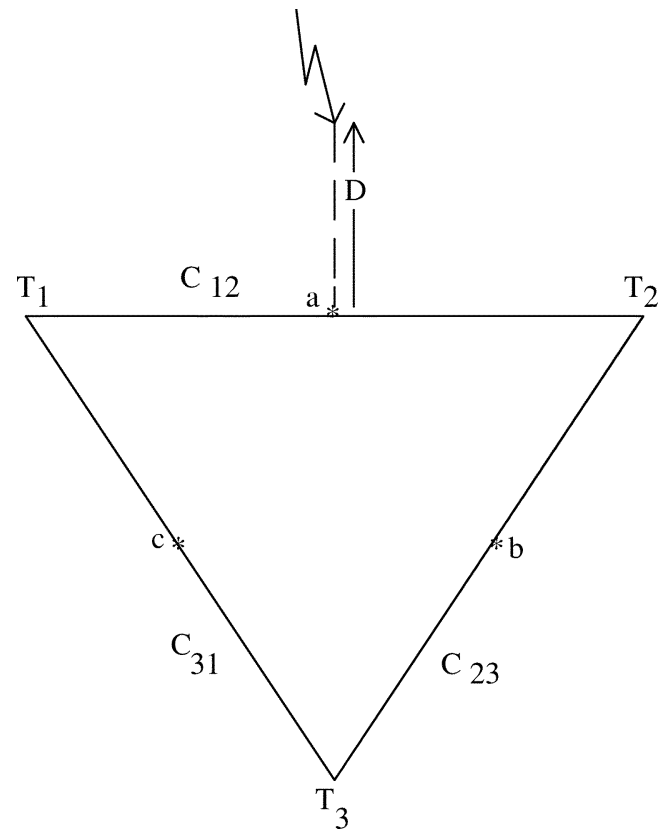

Fig. 4. Sketch showing the protection system and the lightning striking point. $D$ is the striking distance.

the numerical expression for the convolution integral [13] at any node is obtained as follows, for $n=1$

$$
\int_{0}^{\Delta t} \xi(t-\tau) \frac{\partial i}{\partial \tau} d \tau=\xi^{1}(t)\left(i^{1}-i^{0}\right)
$$

for $n>1$

$$
\begin{aligned}
\int_{0}^{n \Delta t} \xi(t-\tau) \frac{\partial i}{\partial \tau} d \tau= & \sum_{m=0}^{m=n-2} \xi^{m+1}(t)\left(i^{n-m}-i^{n-m-1}\right) \\
& +\xi^{1}(t) \frac{\left(i^{n}-i^{n-1}\right)}{2}+\xi^{n}(t) \frac{\left(i^{1}-i^{0}\right)}{2}
\end{aligned}
$$

with $\xi^{k}(t)$ and $i^{k}$ representing the value of the transient ground resistance for the elements in $\left[\xi_{i j}(t)\right]$ and the line current, respectively, at time $t=k \Delta t$

\section{RESULTS AND DisCUSSIONS}

Induced voltages at the conductor midspans and at tower tops due to lightning stroke at distances of 50 and $200 \mathrm{~m}$ have been calculated. Fig. 4 shows the geometry of the protection system and the lightningstrike point. $T_{1}, T_{2}$, and $T_{3}$ are the lightning protection towers, and $C_{12}, C_{23}$, and $C_{31}$ are the conductors interlinking the towers.

In the sketch, five bundled conductors connected between the towers are represented by an equivalent single conductor configuration. The equivalent circuit shown in Fig. 3 may represent any one side of the protection system, eg., the conductor $C_{12}$ connected between the towers $T_{1}$ and $T_{2}$. The towers are represented in the equivalent circuit by a lumped resistance of $420 \Omega$ which is equal to the surge impedance [14] of the tower. The induced voltages are calculated for the striking point distance $D=50$ and $200 \mathrm{~m}$ for a channel base current peak value of $i_{0}=10 \mathrm{kA}$. The results are shown in Figs. 5 and 6 .

Fig. 5 shows the induced voltage at tower tops $T_{1}, T_{2}$, and $T_{3}$ and at the conductormidspans $a, b$, and $c$ assuming the ground as a perfect conductor for a lightning current of $10 \mathrm{kA}$ and a striking distance of $50 \mathrm{~m}$. Because of symmetry, theinduced voltage at towertops $T_{1}$ and $T_{2}$ are same. Similarly for $b$ and $c$, the induced voltages are same. The induced voltage is highest as expected at the conductor midspan $a$ as this is nearer to the lightning striking point. It can be seen that there is a delay introduced in the induced 


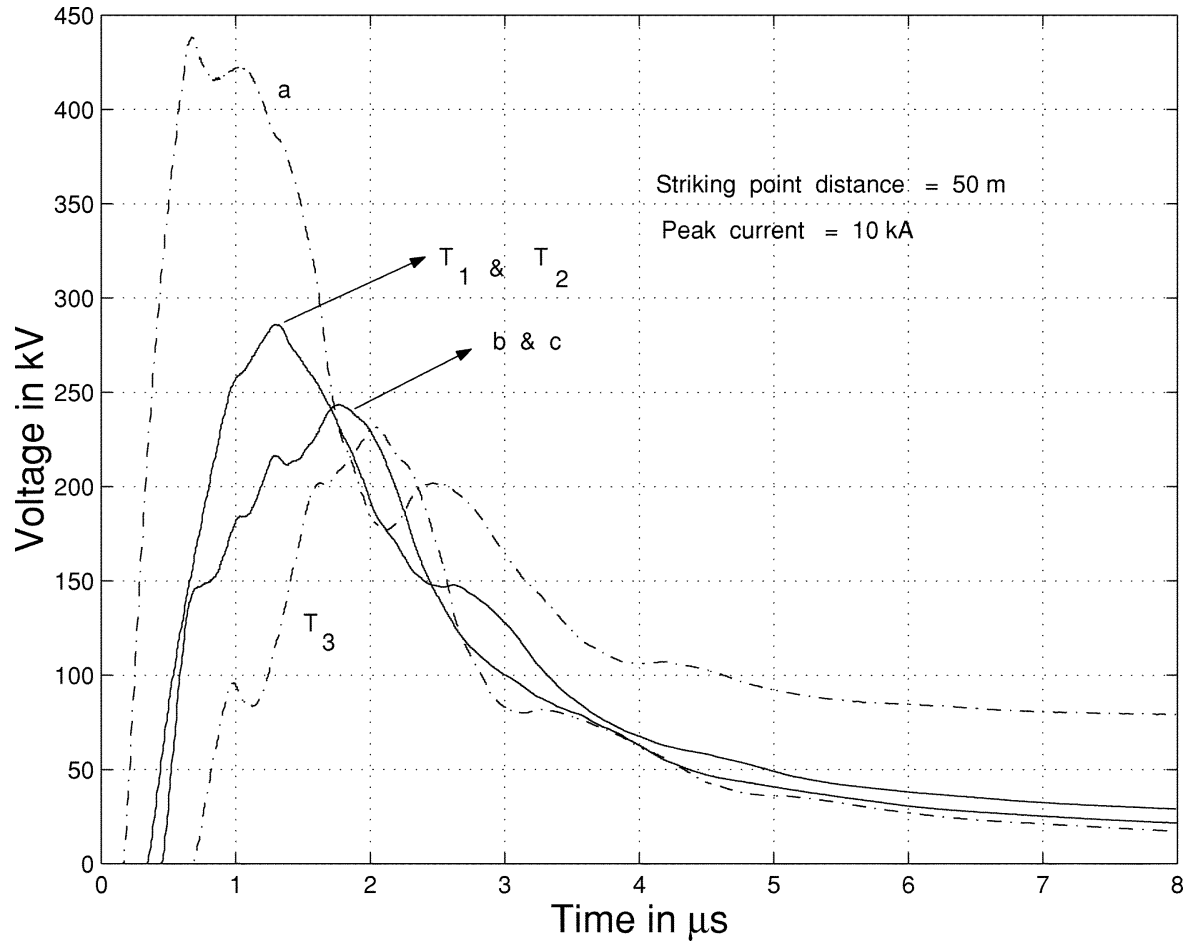

Fig. 5. Induced voltage at tower tops and at conductor midspans assuming ground as a perfect conductor, for a striking distance of $50 \mathrm{~m}$.

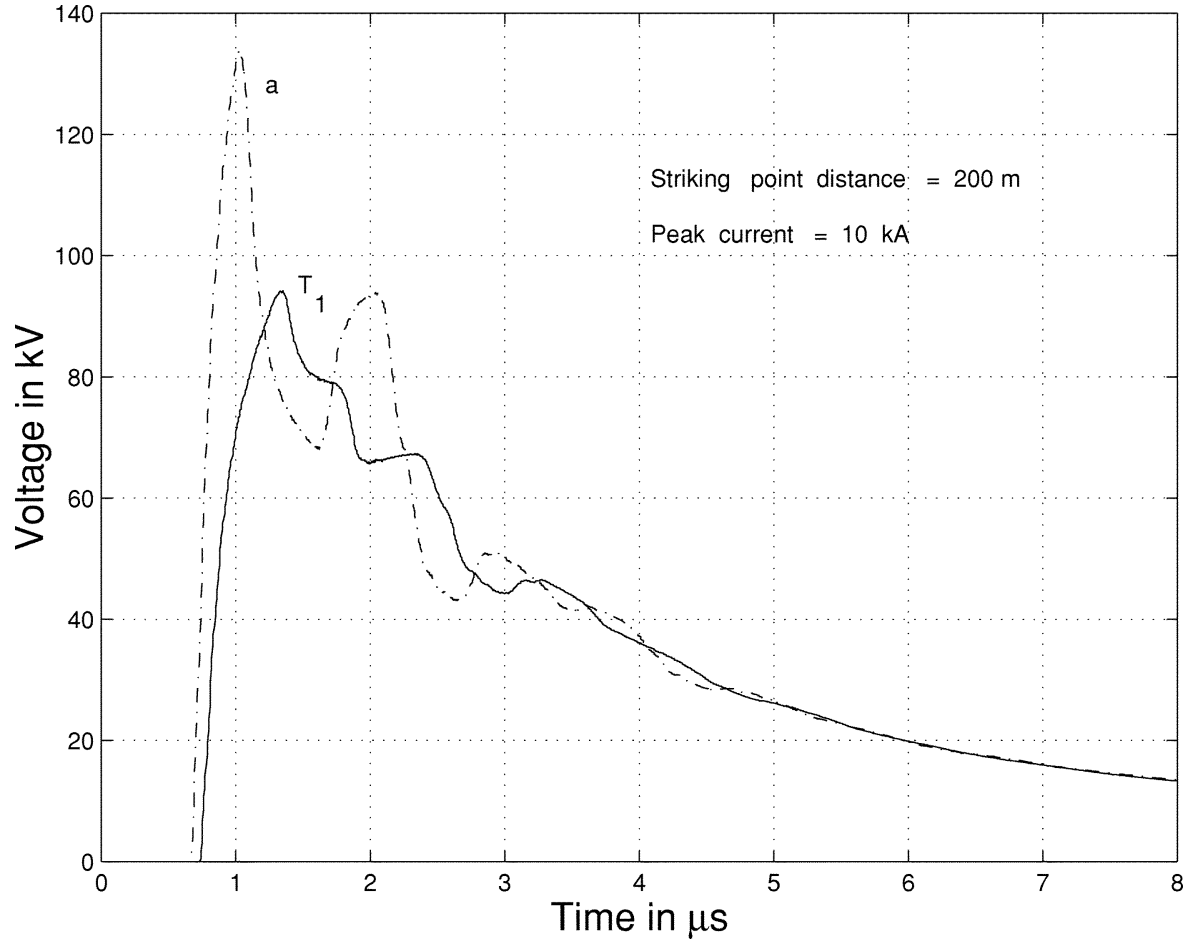

Fig. 6. Induced voltage at top of tower 1 and at conductor midspan $a$ assuming ground as a perfect conductor, for a striking distance of $200 \mathrm{~m}$ and peak current of $10 \mathrm{kA}$.

voltage waveforms. The time delay seen in the waveforms corresponds to the time taken for the electromagnetic field produced by the lightning return stroke to travel to the respective observation points. Fig. 6 shows the waveform of the induced voltage calculated assuming the ground as a perfect conductor for a striking distance of $200 \mathrm{~m}$ and peak current of $10 \mathrm{kA}$. The waveforms are similar to those shown in Fig. 5 except that there are more oscillations in the tail portion of the waveforms as well as a reduction in induced voltages.
The induced voltages have been calculated once again for a peak current of $i_{0}=200 \mathrm{kA}$ and distance of $D=200,500$, and $1000 \mathrm{~m}$ at $a$, the midspan of the conductor $C_{12}$ for the following cases, viz., 1) assuming the ground as a perfect conductor; 2) with a finite ground conductivity of $0.001 \mathrm{~S} / \mathrm{m}$ and relative permittivity of 10.0. A lightning return-stroke peak current of $200 \mathrm{kA}$ has been chosen as this is closest to the highest current level recorded till to-date. For a lightning peak current of $200 \mathrm{kA}$, for any distance chosen less than $200 \mathrm{~m}$ might result 


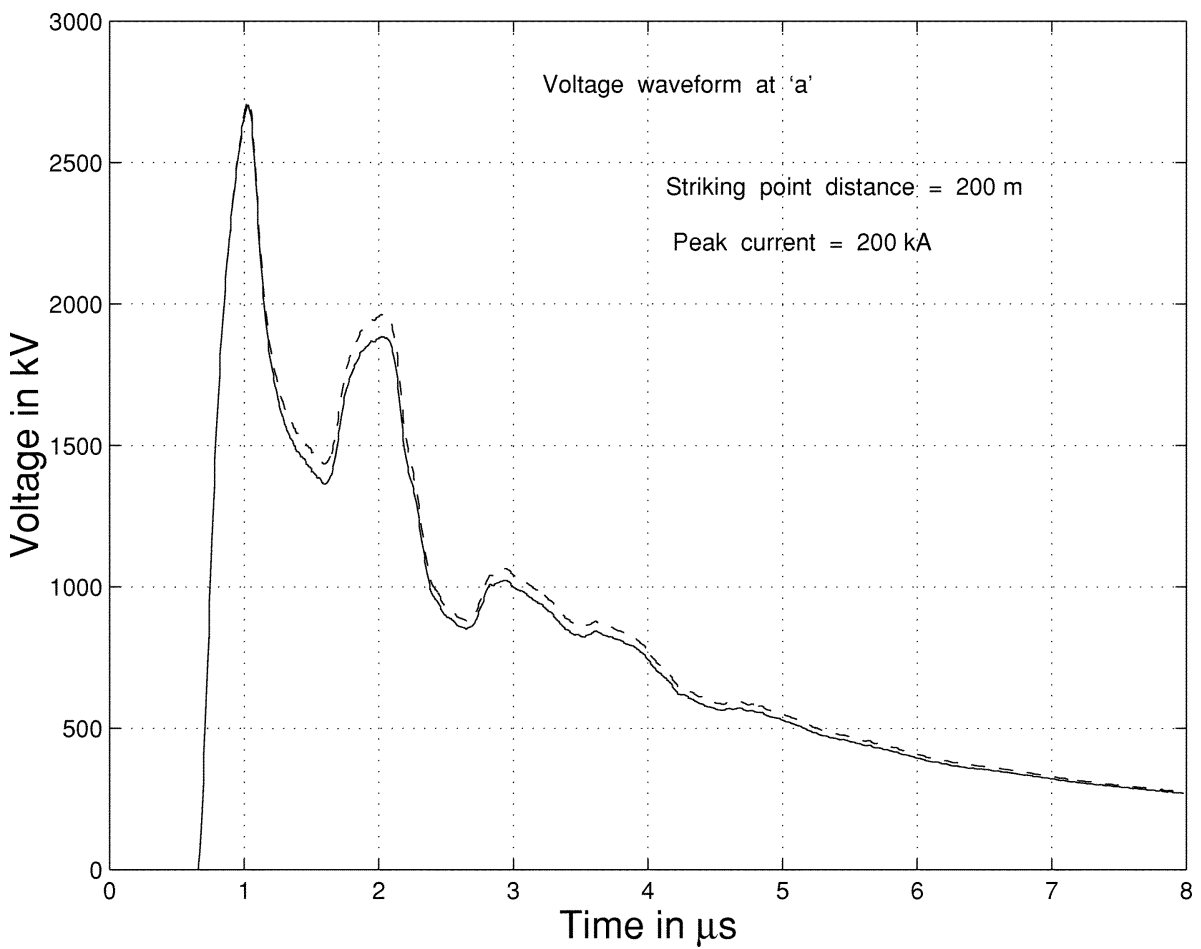

Fig. 7. Induced voltage at $a$. Solid line assuming ground as a perfect conductor, - with ground parameters $\sigma_{g}=0.001 \mathrm{~S} / \mathrm{m}$ and $\epsilon_{r}=10.0$, for a striking distance of $200 \mathrm{~m}$ and peak current of $200 \mathrm{kA}$.

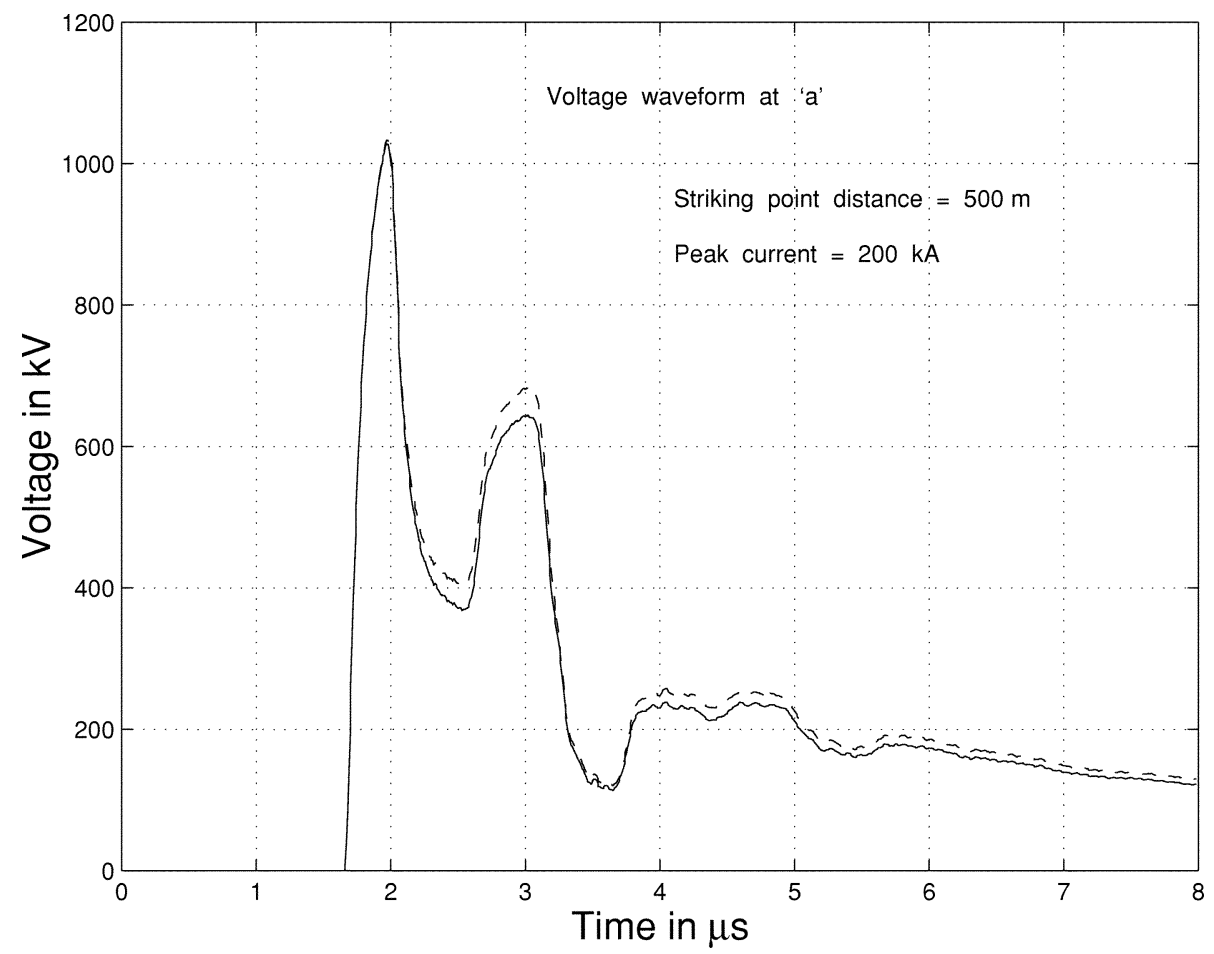

Fig. 8. Induced voltage at $a$. Solid line assuming ground as a perfect conductor, - with ground parameters $\sigma_{g}=0.001 \mathrm{~S} / \mathrm{m}$ and $\epsilon_{r}=10.0$, for a striking distance of $500 \mathrm{~m}$ and peak current of $200 \mathrm{kA}$.

in the stroke terminating on the protection system than on the ground (ie., it becomes a direct strike). Hence, for a peak current of $200 \mathrm{kA}$ computations have been done for distances at and beyond $200 \mathrm{~m}$ only. The voltage waveforms are shown in Figs. 7-9. For higher striking distances, it is seen that the induced voltage becomes more distorted for the same channel base peak current. For $200 \mathrm{kA}$ at a striking distance of $200 \mathrm{~m}$, the induced voltage could reach as high as $2.7 \mathrm{MV}$ which could be comparable to the voltage developed due to a direct lightning stroke of lower peak current. However, the waveshape will be entirely different [1]. It is seen from the figures that the ground conductivity has an insignificant influence on the magnitude as well as the waveshape of the induced voltage. This is in contrast to where the ground 


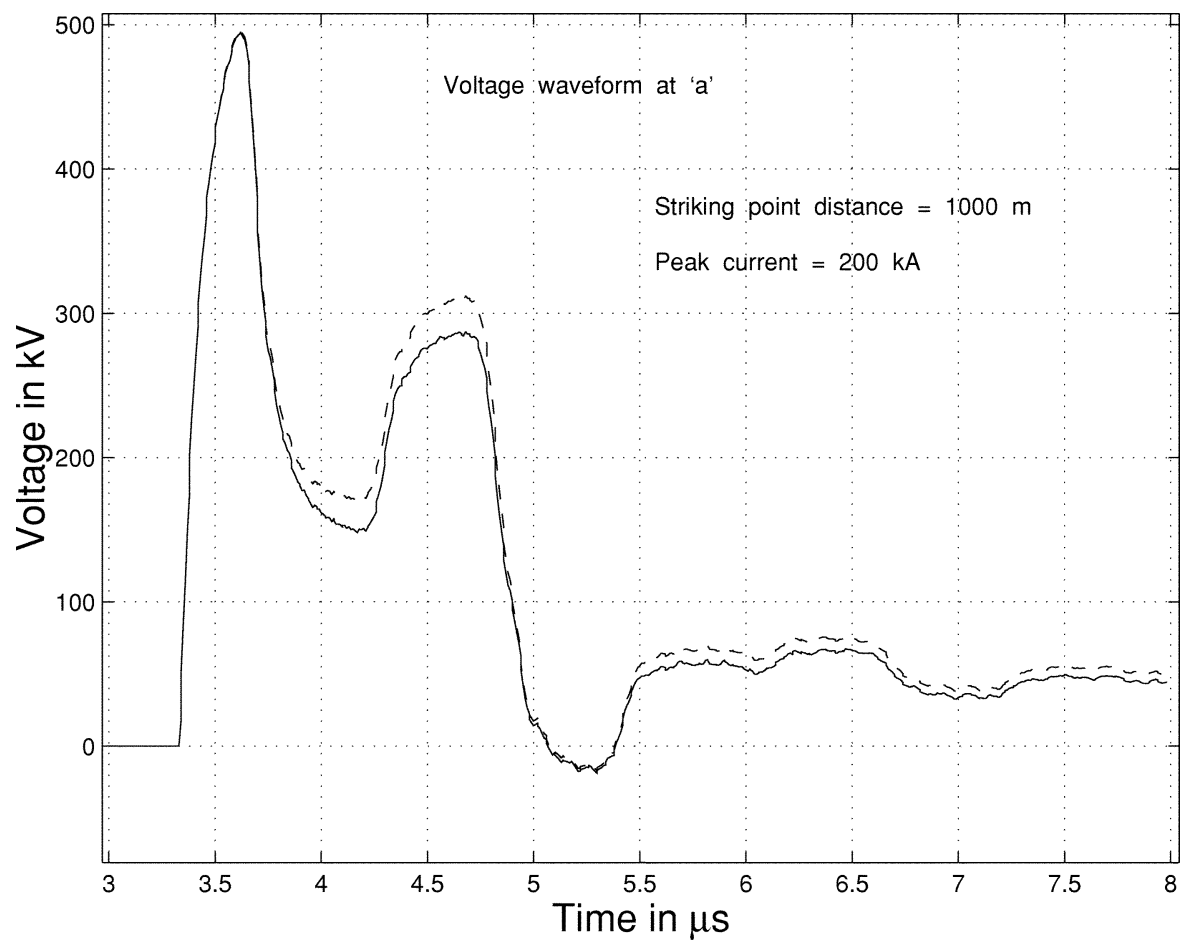

Fig. 9. Induced voltage at $a$. solid line assuming ground as perfect conductor, - with ground parameters $\sigma_{g}=0.001 \mathrm{~S} / \mathrm{m}$ and $\epsilon_{\mathrm{r}}=10.0$, for a striking distance of $1000 \mathrm{~m}$ and peak current of $200 \mathrm{kA}$.

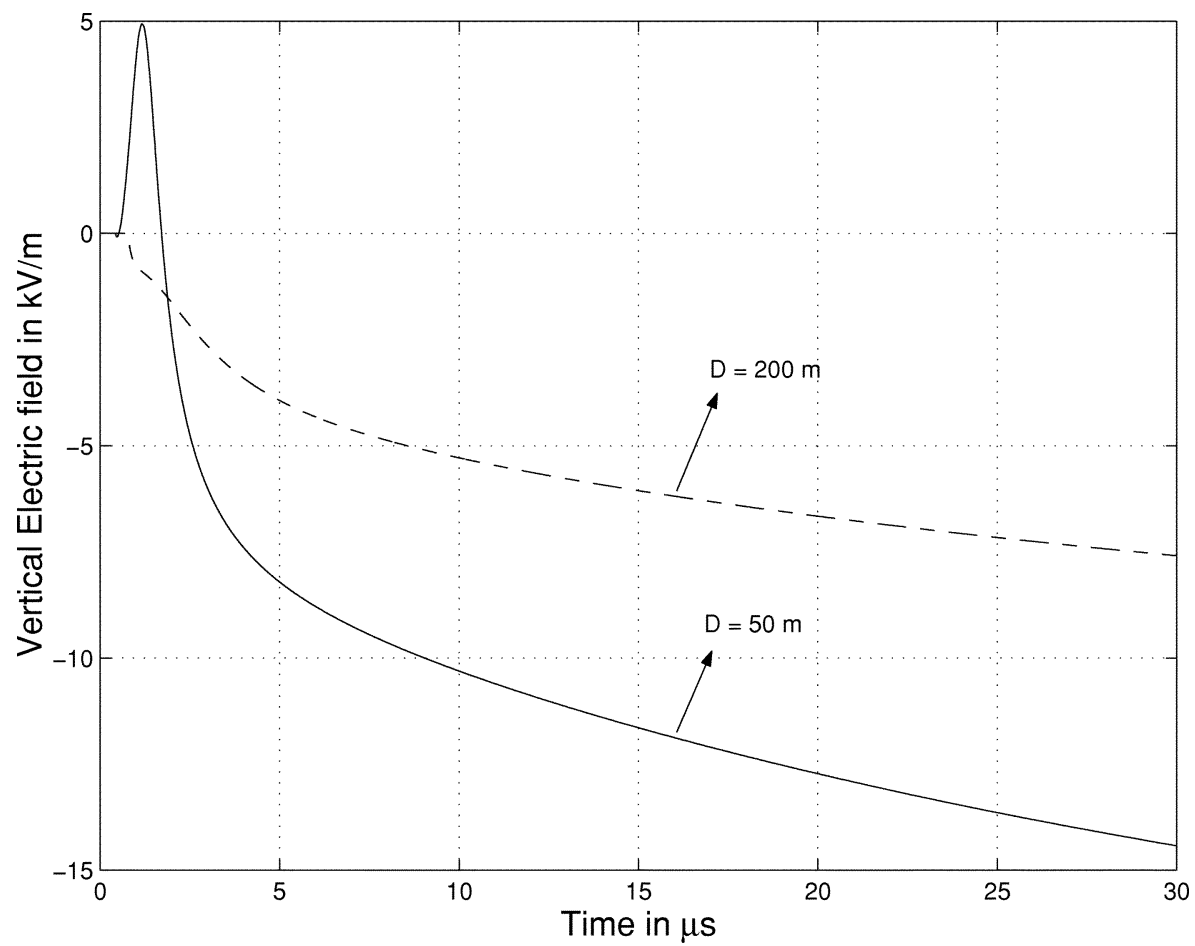

Fig. 10. Vertical electric field at a height of $120 \mathrm{~m}$ for the peak current of $10 \mathrm{kA}$.

conductivity has a definite influence on the magnitude and waveshape of the induced voltage on the conductors which are few meters above the ground, eg., the power distribution line [15].

In general, for an overhead conductor of long length at a short height, when Agrawal's coupling model is used as in this study, the magnitude and waveshape of the induced voltage mainly depends on the horizontal component of the electric field. However, for conductors which are at large height and of a short length, this is not true.

When finite ground conductivity is taken into account, the horizontal electric field is the difference of horizontal electric field calculated assuming ground as a perfect conductor and a term involving magnetic field at ground level. Now, for large heights, the contribution from the term containing the magnetic field is negligible compared to the first 


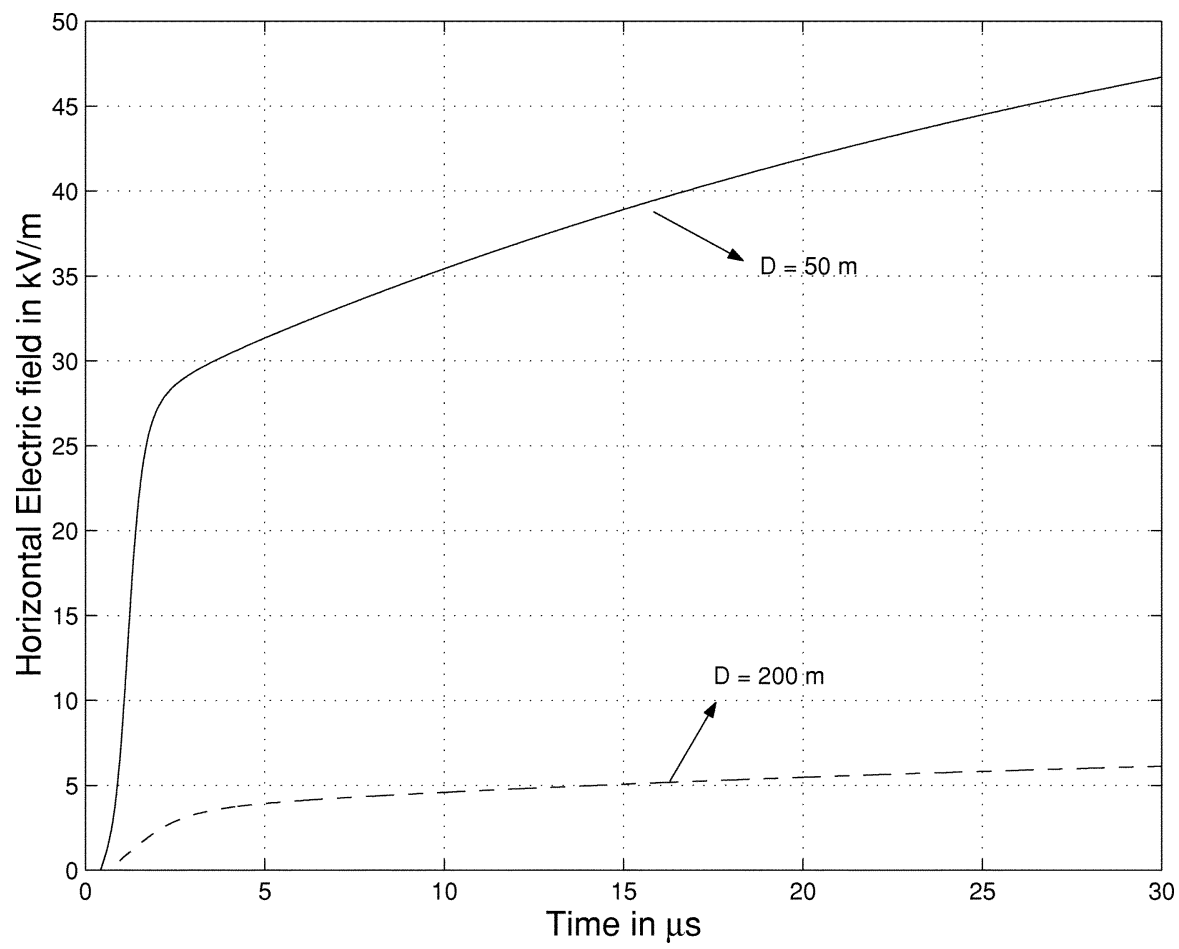

Fig. 11. Horizontal electric field at a height of $120 \mathrm{~m}$ assuming the ground as a perfect conductor, for the peak current of $10 \mathrm{kA}$.

term which is nothing but the horizontal electric field assuming ground as a perfect conductor. Hence for large heights, the horizontal electric field with a finite conductivity of ground is nearly equal to that of horizontal electric field calculated assuming ground as a perfect conductor Moreover, within the Agrawal et al. coupling model, the contribution of vertical electric field on induced voltage is more than the contribution due to horizontal electric field for this structure. Because of the large heights involved, the vertical electric field has been computed at different heights and the induced voltage is found by integrating the vertical electric field along the height. For such large heights, the contribution on the induced voltage due to the vertical electric field (which is not influenced by the ground conductivity) is more than that of the contribution due to horizontal electric field. Hence, the influence of finite ground conductivity on the total induced voltage is negligibly small.

From Figs. 5-9, it can be also observed that the oscillation in the induced voltage waveform is more for large striking distances. The vertical and horizontal electric field calculated for a base current of $10 \mathrm{kA}$ and at a tower height of $120 \mathrm{~m}$ for striking distances of 50 and $200 \mathrm{~m}$ are shown in Figs. 10 and 11. It is seen from the figures that the decrease in the magnitudes of these fields with increase in striking distance are not in the same proportion. This in turn may contribute to the distortion in waveshape of the induced voltages for different lightning striking distances.

\section{CONCLUSION}

The induced voltage at the tower top and midspan of the conductors connecting the towers of a lightning protection system due to a nearby lightning return stroke has been calculated. It has been observed that the waveshape of the induced voltage depends on conductor height and striking distance and that the oscillation in the induced voltage is more for large striking distance. The ground conductivity has negligible effect on the magnitude and waveshape of the induced voltage unlike for power distribution line conductors which are at few meters above the ground.

\section{ACKNOWLEDGMENT}

The authors wish to thank the authorities of Indian Institute of Science, Bangalore, India, for the permission to publish this paper; Prof C. A. Nucci, University of Bologna, Bologna, Italy for the valuable discussions during his visit to Department of High Voltage Engineering, Indian Institute of Science. The first author would like to thank the Ministry of Human Resources Development, Goernment of India, and the National Institute of Technology of Karnataka, Mangalore, India for deputing him for taking up the research on the above topic.

\section{REFERENCES}

[1] M. J. Thomas and G. R. Nagabhushana, "Development of a Lightning Protection Scheme for Satellite Launch Pad at Sriharikota," Indian Inst. Sci., Bangalore, India, ISRO Rep., 1997.

[2] C. A. Nucci, "Lightning-induced voltages on overhead power lines-part I," Electra, no. 161, pp. 75-102, 1995.

[3] A. Karwowski and A. Zeddam, "Transient currents on lightning protection system due to indirect lightning effect," Proc. IEE Sci. Meas. Technol., vol. 142, no. 3, pp. 213-222, 1995.

[4] C. A. Nucci, F. Rachidi, M. Ianoz, and C. Mazzetti, "Lightning-induced voltages on overhead lines," IEEE Trans. Electromagn. Compat, vol. 35 pp. 75-85, Feb. 1993.

[5] M. A. Uman, "Lightning return stroke electric and magnetic fields," $J$. Geophy.Res, vol. 90, no. D4, pp. 6121-6130, June 1985.

[6] M. J. Master and M. A. Uman, "Lightning induced voltages on power lines: theory," IEEE Trans. Power App. Syst, vol. PAS-103, pp. 2502-2518, Sept. 1984

[7] M. Rubinstein, "An approximate formula for the calculation of the horizontal electric field from lightning at close, intermediate and long ranges," IEEE Trans Electromagn. Compat., vol. 38, pp. 531-535, Aug. 1996.

[8] A. K. Agrawal, H. J. Price, and S. J. Gurbaxani, "Transient response of multiconductor transmission lines excited by a nonuniform electromagnetic field," IEEE Trans Electromagn. Compat., vol. 22, pp. 119-129, May 1980.

[9] F. Rachidi, C. A. Nucci, M. Ianoz, and C. Mazzetti, "Influence of lossy ground on lightning induced voltages on overhead lines," IEEE Trans Electromagn. Compat., vol. 38, pp. 250-264, Aug. 1996. 
[10] D. Orzan, "Time-domain low-frequency approximation for off-diagonal terms of the ground impedance matrix," IEEE Trans. Electromagn. Compat., vol. 39, p. 64, Feb. 1997.

[11] F. Rachidi, C. A. Nucci, and M. Ianoz, "Transient analysis of multiconductor lines above a lossy ground," IEEE Trans. Power Delivery, vol. 14, pp. 294-302, Jan. 1999.

[12] S. L. Loyka, "On calculation of the ground transient resistance of overhead lines," IEEE Trans. Electromagn. Compat., vol. 41, pp. 193-195, Aug. 1999.

[13] E. O. Brigham, The Fast Fourier Transform. Englewood Cliffs, NJ: Prentice-Hall, 1974.

[14] F. S. Costa and G. C. Miranda, "Comparison of lightning tower surge models using the EMTP," in Proc.10th Int. Symp. High Voltage Engineering, Montreal, Canada, Aug. 1997, pp. 327-330.

[15] P. D.P. Durai Kannu and M. J.M. Joy Thomas, "Influence of ground conductivity on the over voltages induced on overhead power distribution lines due to an indirect lightning stroke," in Proc. IEEE Int. Symp. Electromagnetic Compatibility, vol. 2, Washington, DC, 2000, pp. 949-954

\section{Numerical Electromagnetic Analysis of Lightning-Induced Voltage Over Ground of Finite Conductivity}

\author{
Ramesh K. Pokharel, Masaru Ishii, and Yoshihiro Baba
}

\begin{abstract}
Numerical Electromagnetics Code (NEC-2), which is a computer code to analyze the three-dimensional electromagnetic (EM) field around thin wires in the frequency domain, has been successfully used in lightning-related studies such as lightning surge analyses or lightning EM pulse calculations over a perfectly conducting ground. NEC-2 is still more useful in investigating lightning-induced effects over ground of finite conductivity. This application of NEC-2 is investigated by comparing calculated results with an experiment over lossy ground. The advantages of the analysis using NEC-2 are that it can accurately compute the current distribution along a wire structure with small amount of postulation. It automatically incorporates coupling between a lightning channel and transmission lines, and it can also take account of finitely conducting ground in transient condition. In addition, it can also model conductors in any arbitrary angles, and it is available in the public domain.
\end{abstract}

Index Terms-Ground conductivity, lightning channel, lightning electromagnetic pulse (LEMP), lightning-induced voltage, numerical EM analysis.

\section{INTRODUCTION}

It is important to predict lightning-induced voltage on overhead wires to protect distribution lines or communications lines against lightning. In the study of lightning-induced voltages, modeling of coupling of an overhead wire with a lightning channel has been one of the most important issues [1]-[6]. Influence of finite conductivity of ground and modeling of a lightning channel are other factors of primary importance in evaluating lightning-induced voltages.

One of the earliest applications of a numerical electromagnetic (EM) analysis to lightning study [7] modeled a lightning channel attached to

Manuscript received October 15, 2002; revised January 7, 2003.

R. K. Pokharel and M. Ishii are with the Department of Electrical Engineering, the University of Tokyo, Tokyo 153-8505, Japan (e-mail: pokharel@ iis.u-tokyo.ac.jp; ishii@iis.u-tokyo.ac.jp).

Y. Baba is with the Department of Electrical Engineering, Doshisha University, Kyoto 610-0321, Japan (e-mail: ybaba@mail.doshisha.ac.jp).

Digital Object Identifier 10.1109/TEMC.2003.819065 a tall structure. A time-domain code was used in that study. A similar time-domain code was also used in a study on induced voltages on a distribution line [8] over perfectly conducting ground. A recent study also employs a similar time-domain code [9] to model a lightning channel [10]; however, more authors have applied the Numerical Electromagnetics Code (NEC-2) [11], a frequency-domain code, to lightning electromagnetic pulse (LEMP) studies or lightning-surge analysis [12]-[17]. Heidler et al. [12] used NEC-2 to analyze induced voltages on conductor loops illuminated by LEMP. Cristina et al. [13] employed it to evaluate the EM field inside a building struck by lightning. Chai et al. [14] employed this code to study field inside a wire-array lightning protection system for a launch vehicle when it is struck by lightning. The last two authors of this paper have also applied this code to analyze transient voltages across an insulator of a power transmission tower struck by a direct lightning [15], [16].

In this paper, the accuracy of NEC-2 in evaluating lightning-induced voltages over lossy ground is discussed by comparison with the results obtained by an experiment on a reduced-scale model and with another method [18], which employs Agrawal's coupling model [19] and Norton's approximation [20] to evaluate fields over ground of finite conductivity. The comparison of the computed results shows that NEC-2 can practically be applied to analyze the lightning-induced voltages on a distribution line over ground of finite conductivity.

\section{Method OF ANALYSIS}

NEC-2 solves the boundary value problem of the electric field integral equation in the frequency domain by the method of moments [21]. It is particularly effective to analyze the EM response of antennas or other metallic structures composed of thin straight wires. For numerical analysis, it requires that the entire structure to be divided into cylindrical segments that should be shorter than $1 / 10$ of the wavelength of interest [11]. Once the model is defined, the excitation is imposed as a voltage source or a plane wave. Current on each segment is calculated as the output. To solve problems in the time domain, the fast Fourier transform (FFT) of the excitation and inverse FFT of the outputs are necessary. Examples of application of NEC-2 to time-domain problems of power engineering were demonstrated by Ishii and Baba [22].

NEC-2 permits each element to have impedance. In calculating line voltages, an element having high resistance such as $10^{7} \Omega$ is inserted between the line and ground, and the voltage across the element is calculated from the induced current on it.

In evaluating lightning-induced voltages or LEMP study by NEC-2, an EM model is employed to represent a return stroke [23]. In an EM model, a lightning channel is modeled by a thin wire, and it is either surrounded by a medium of high permittivity [10], or contains distributed series resistance and additional inductance [17] to reproduce the reduced propagation velocity of channel current. The latter approach is used in this paper.

In calculating the EM field above the finitely conducting ground, NEC-2 has an option employing Norton's approximation [20] and numerical solution of Sommerfeld integral [24]. The accuracy of the calculation over finitely conducting ground, however, has not been verified yet. Therefore, not only to demonstrate the ability of the proposed numerical method in coping with the coupling problem, but also to investigate the accuracy of the option of NEC-2, comparison is made between calculated and experimental results of induced voltages over finitely conducting ground. 